Tropical Journal of Pharmaceutical Research September 2019; 18 (9): 1823-1829

ISSN: 1596-5996 (print); 1596-9827 (electronic)

(1) Pharmacotherapy Group, Faculty of Pharmacy, University of Benin, Benin City, 300001 Nigeria.

\title{
MicroRNA-187 inhibits pentylenetetrazol-induced neuronal apoptosis and alleviates development of epilepsy in epileptic rats by regulating SPRY1 expression
}

\author{
Limei Diao, ${ }^{1,2}$, Haichun $\mathrm{Yu}^{3}$, Shungui Wang ${ }^{4}$, Qian $\mathrm{Yu}^{4}$, Huaxia $\mathrm{Li}^{4}$, Ling $\mathrm{Lu}^{4}$, \\ Xianqiu Liao ${ }^{4}$, Huan $\mathrm{Li}^{4}$, Xiaoqiang Qiu ${ }^{1 *}$ \\ ${ }^{1}$ Department of School of Public Health, Guangxi Medical University, Nanning, Guangxi, 530021, ${ }^{2}$ Department of Neurology, \\ The First Affiliated Hospital of Guangxi University of Chinese Medicine, Nanning, Guangxi 530023, ${ }^{3}$ Department of Numerical \\ Control Technology, Guangxi Technological College of Machinery and Electricity, Nanning, Guangxi 530007, ${ }^{4}$ Department of \\ Neurology, Graduate School of Guangxi University of Chinese Medicine, Nanning, Guangxi 530023, China
}

*For correspondence: Email: QiuXQiangcbn@163.com; Tel: +86-771-5350823

\begin{abstract}
Purpose: To explore the role of microRNA-187 on the pathological process of epilepsy. Methods: The seizure score of epileptic rats was evaluated according to Racine's scale. Real-time quantitative polymerase chain reaction (RT-qPCR) was performed to determine the expression levels of microRNA-187 (miR-187). Western blot technique was conducted to assess the expression levels of caspase 3, B-cell lymphoma-2 (BCL-2), and poly (ADP-ribose) polymerase (PARP)] and activation of phosphatase and tensin homolog (PTEN)/PI3K/AKT cascade. Caspase 3 colorimetric assay kit was employed to evaluate the activity of caspase 3. Dual-luciferase reporter gene system was used to explore the regulating mechanisms of miR-187 and protein sprouty homolog 1 gene (SPRY1).

Results: The results showed that miR-187 was aberrantly downregulated in the hippocampus regions of pentylenetetrazol (PTZ)-treated rats compared to normal rats $(p<0.05)$. Furthermore, PTZ promoted caspase 3-dependent neuronal apoptosis by increasing the expression of pro-apoptosis protein PARP and decreasing the expression levels of BCL-2 in rats. On the other hand, overexpression of miR-187 downregulated SPRY1 as well as PTEN ( $p<0.05)$, thereby activating the downstream PI3K/AKT signaling pathway. Notably, the effects of upregulated miR-187 on neuronal apoptosis and epilepsy development in PTZ-induced rats was reversed by the concomitant overexpression of SPRY1 ( $p<$ 0.05).

Conclusion: The results of this research show that overexpressed miR-187 alleviates the development of PTZ-induced neuronal apoptosis and epilepsy in epileptic rat models by regulating SPRY1 expression. These findings can hopefully be beneficial for the discovery of new therapeutic strategies for epilepsy treatment.
\end{abstract}

Keywords: Epilepsy, Neuronal apoptosis, miR-187, SPRY1

This is an Open Access article that uses a fund-ing model which does not charge readers or their institutions for access and distributed under the terms of the Creative Commons Attribution License (http://creativecommons.org/licenses/by/4.0) and the Budapest Open Access Initiative (http://www.budapestopenaccessinitiative.org/read), which permit unrestricted use, distribution, and reproduction in any medium, provided the original work is properly credited.

Tropical Journal of Pharmaceutical Research is indexed by Science Citation Index (SciSearch), Scopus, International Pharmaceutical Abstract, Chemical Abstracts, Embase, Index Copernicus, EBSCO, African Index Medicus, JournalSeek, Journal Citation Reports/Science Edition, Directory of Open Access Journals (DOAJ), African Journal Online, Bioline International, Open-J-Gate and Pharmacy Abstracts 


\section{INTRODUCTION}

Epilepsy seriously endangers the human health worldwide and degrades the life quality of human beings to a large extent [1]. Currently, there are several methods for epilepsy treatment and monitoring that help alleviate the symptoms of patients with epilepsy to some extent [2]. However, the therapeutic effects of these treatment methods are limited due to a lack of adequate knowledge regarding the underlying mechanisms of epilepsy development.

Neuronal apoptosis is closely associated with epilepsy, and targeting neuronal apoptosis has been shown to be a potential therapeutic method [3]. Nevertheless, the underlying mechanisms involved in regulating neuronal apoptosis in epilepsy are still not fully delineated.

Small non-coding RNA microRNA (miRNA) contains approximately 22 nucleotides on average [4], functions as a gene expression regulator, and participates in the pathogenesis of multiple diseases. Recently, miRNA was reported to be closely related to neurodegenerative disorders and the development of epilepsy [5]. For example, suppression of miR-141 has been shown to protect against neuronal apoptosis in epilepsy [6]. Targeting of miR-21-5p protects rats against seizure damage by regulating the phosphatase and tensin homolog (PTEN)-mTOR signal pathway [7]. Also, miR-187 participated in the regulation of multiple diseases pathogenesis and progression [8].

In addition, by analyzing the miRNA expression profile of GSE49850, miR-187 was identified as playing a critical role in the pathogenesis of epilepsy [9]. Furthermore, the levels of miR-187 were closely related with epilepsy progression in both epileptic rat models and patients with temporal lobe epilepsy [10]. In spite of these reports and findings, the regulatory role of miR187 in epilepsy development remains still unclear. Uncovering the underlying mechanisms might be helpful for the discovery of novel therapeutic agents for epilepsy treatment in clinic.

Protein sprouty homolog 1 gene (SPRY1) was crucial for regulating cell proliferation, migration, apoptosis and neurological functions [11]. In addition, sprouty homolog 1 is the downstream target of several miRNAs [12]. Hence this study aimed at investigating the role of miR-187SPRY1 interaction in the development of PTZinduced neuronal apoptosis and epilepsy.

\section{EXPERIMENTAL}

\section{Establishment of animal models}

Adult male rats weighing 260 to $300 \mathrm{~g}$ were maintained under standard experimental conditions of temperature of $25^{\circ} \mathrm{C}$, and humidity of $50-60 \%$. Animal feed and water were available ad libitum. The animal experiments were in compliance with the Guidelines for the Care and Use of Laboratory Animals" of the Institutional Animal Care and Use Committees (IACUCs) of the United States [24]. All animal studies were approved by the Animal Care and Use Committee of Guangxi Medical University (Approval Number: 2018-038-02). Pentylenetetrazol (PTZ) dissolved in artificial cerebrospinal fluid (ACSF) was administered according to the body weights of the rats (30 $\mathrm{mg} / \mathrm{kg}$ ) on alternate days for a maximum of 35 days. Then, each rat was placed in an isolated transparent box and observed individually for 30 min. Racine's scale was used to evaluate the seizure score: $0=$ no response; $1=$ ear and facial cramping; 2 = myoclonic jerk without rearing; $3=$ myoclonic jerk with rearing; $4=$ change into the side position (e.g., clonic-tonic seizures); and $5=$ change into the supine position, including generalized tonic-clonic convulsions.

\section{Real-time quantitative polymerase chain reaction (RT-qPCR)}

After treating rats with PTZ and successfully establishing the epileptic model, the hippocampus tissues of the rats were isolated and the TRIzol kit (Invitrogen Life Technologies, Carlsbad, California, USA) was employed to extract total RNA from the tissues. Reversetranscription PCR analysis by iScript cDNA Synthesis Kit (Bio-rad, Hercules, CA, USA) and RT-qPCR analysis by HiScript II Q Select RT SuperMix (Vazyme Biotech Co. Ltd., Nanjing City, China) were purchased to evaluate gene expression. The primers of these genes are designed and listed in Table 1.

\section{Western blot}

The Western Blot technique was performed according to the previous studies. Briefly, Total proteins were acquired from the hippocampus tissues of the rats with or without PTZ treatment by using the RIPA lysis buffer purchased from Beyotime (Jiangsu, China). The bicinchoninic acid (BCA) kit (Pierce, Rockford, IL) was utilized to quantify the total proteins, which were then separated by using $10 \%$ SDS/PAGE at $80 \mathrm{~V}$ for $2 \mathrm{~h}$ at $4{ }^{\circ} \mathrm{C}$ and transferred to polyvinylidene 
difluoride (PVDF) membranes (Millipore, Bedford, MA).

Table 1: Quantitative PCR primer sequences

\begin{tabular}{ll}
\hline Gene & Primer sequence (strand) \\
\hline 3-actin & Forward: 5'- \\
& CTCCATCCTGGCCTCGCTGT-3' \\
& Reverse: 5'- \\
& GCTGCTACCTTCACCGTTCC-3' \\
U6 & Forward: 5'-CTCGCTTCGGCAGCACA-3' \\
& Reverse: 5'- \\
& AACGCTTCACGAATTTGCGT-3' \\
miR-187 & Forward: 5'- \\
& GGCUACAACACAGGACCCGGGC-3' \\
& Reverse: 5'- \\
& GCCCGGGUCCUGUGUUGUAGCC-3' \\
SPRY1 & Forward: 5'- \\
& GCCTTCTTTGGATAGCCGTCAG-3' \\
& Reverse: 5'- \\
& TCATTGCTGCCTCTTATGGCC-3' \\
\hline
\end{tabular}

The PVDF membranes were probed with the antibodies including SPRY1 (1:1000, \#ab75492, Abcam, Cambridge, UK), cleaved caspase 3 (1:1000, \#ab32351, Abcam), GAPDH (1:5000, \#ab181602, Abcam), PARP (1:500, \#ab74290, Abcam), BCL-2 (1:1000, \#ab32124, Abcam), PTEN (1:1000, \#ab32199, Abcam), PI3K (1:1000, \#ab32089, Abcam), AKT (1:1000, ab8805, Abcam) and p-AKT (1:1000, \#ab81283, Abcam) overnight at $4{ }^{\circ} \mathrm{C}$. After washing with TBST buffer (a mixture of Tris-buffered saline and Tween), the membranes were then incubated with the secondary antibody (1:5000, Santa Cruz Blotechnology, CA, USA) for $1 \mathrm{~h}$ at room temperature. The protein bands were then quantified by using a ECL Western Blot system purchased from Thermo Fisher Scientific (MA, USA).

\section{Dual-luciferase reporter gene system}

The fragments of human SPRY1 gene containing the binding sites were cloned into the pGL3control vector by Ambion (Austin, TX) to generate SPRY1-WT vectors. The binding sites in in SPRY were then mutated by using a QuickChange site-directed Mutagenesis Kit purchased from Agilent Technologies (CA, USA) to generate SPRY1-MUT vectors.

Next, 293T cells were cultured under standard conditions and the above vectors were transfected into the cells. The Dual-Luciferase Reporter Gene System (Promega, Madison, WI, USA) was utilized to evaluate the luciferase activities of Firefly and Renilla after $48 \mathrm{~h}$ of transfection, which represented the regulating relationship between miR-187 and SPRY1.

\section{Determination of caspase 3 activity}

Tissues from the hippocampus of PTZ-treated rats were collected, and caspase 3 activity was evaluated by using a caspase 3 colorimetric assay kit purchased from Abcam (MA, USA). In brief, the tissues were collected, the lysis buffer solution was used to resuspend the above tissues. After that, the tissues were placed into the lysis buffer and a BCA kit (Pierce, IL, USA) was used to quantify the total proteins, and the supernatants were collected. Finally, the reaction buffer was then used to treat the supernatants and the activity of Caspase 3 was determined at the absorbance of $405 \mathrm{~nm}$.

\section{Statistical analysis}

The data were collected and presented as mean \pm standard deviation (SD) and analysed by SPSS 18.0 software. The Student's $t$ test for the comparison between two groups, and the oneway analysis of variance (ANOVA) for the comparisons among multiple groups. $P<0.05$ means statistical significance.

\section{RESULTS}

\section{Aberrant expression of $\mathrm{miR}-187$ in the hippocampus tissues of PTZ-treated rats}

To investigate the association between miR-187 and the development of epilepsy in rats, PTZ was used to establish epileptic rat models. The results showed that the epileptic rat models were successfully established based on the seizure score (Figure $1 \mathrm{~A}$ ). In addition, the RT-qPCR results showed that $\mathrm{miR}-187$ significantly downregulated in the hippocampus tissues of PTZ-treated rats compared to the control group (Figure $1 \mathrm{~B}$ ).
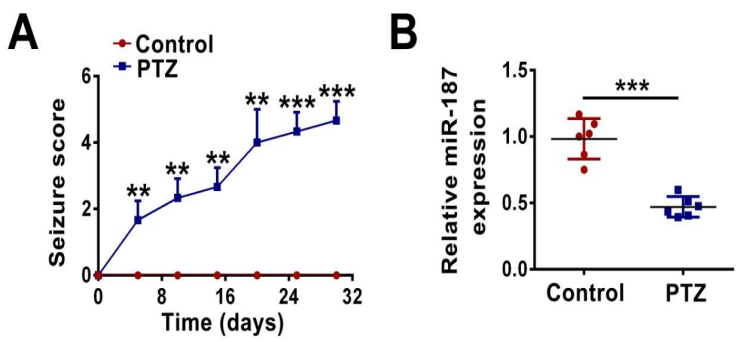

Figure 1: The expression of miR-137 in the hippocampus tissues of PTZ-treated rats. (A) Seizure score of epileptic rats evaluated according to the Racine's scale. (B) miR-187 level was evaluated by RT-qPCR in the hippocampus of epileptic rats $(n=6)$; ${ }^{* \star} p<0.05,{ }^{* * *} p<0.01$ 
Effect of overexpressed miR-187 on neuronal apoptosis in PTZ-treated rat models

Based on the above findings, this study next explored the functions of miR-187 in PTZinduced epileptic rats. Surprisingly, the results shows that the development of PTZ-induced epilepsy in rats was attenuated by overexpression of miR-187 (Figure $2 \mathrm{~A}$ ), which indicates that miR-187 might be a potential therapeutic agent for epilepsy. In addition, PTZ treatment downregulated miR-187 level and improved the activity of caspase 3 in neuronal cells in rat hippocampus, which were reversed by the concomitant overexpression of miR-187 (Figure $2 \mathrm{~B}$ and $\mathrm{C}$ ). The expression levels of BCL-2 were downregulated, whereas the expressions of caspase 3 and PARP were upregulated by PTZ treatment in rat neuronal cells. The expressional changes of the above proteins were also reversed by the concomitant overexpression of miR-187 (Figure 2 D).

A
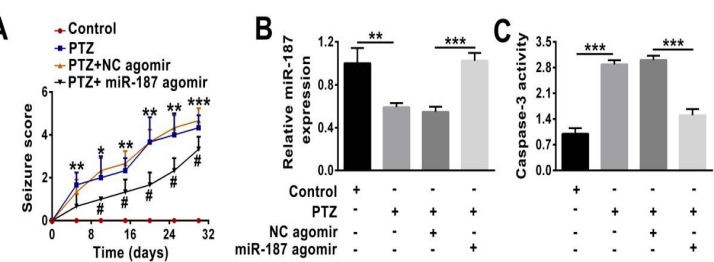

D
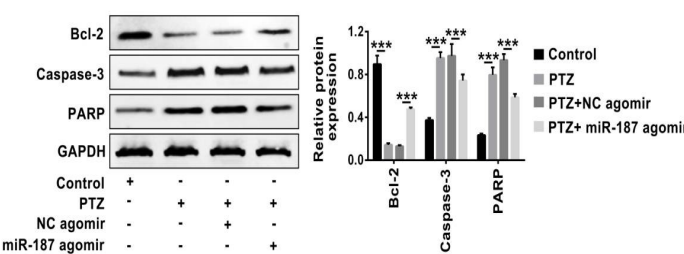

Figure 2: Influence of miR-187 on neuronal apoptosis. (A) Seizure scores of epileptic rats with or without miR-187 agomir treatment were evaluated according to the Racine's scale $(n=6)$. (B) miR-187 expression was evaluated by RT-qPCR in the hippocampus of epileptic rats. (C) Caspase 3 activity was determined by using the caspase 3 Activity Assay Kit. (D) The expression levels of BCL-2, caspase 3, and PARP were evaluated by Western Blot, normalized by GAPDH. ${ }^{* *} p<0.05,{ }^{* * *} p<0.01$, compared to control group

\section{SPRY1 and its downstream PTEN/PI3K/AKT signal pathway are potential targets of miR- 187}

TargetScan software results showed that the 3' UTR region of SPRY1 has miR-187 binding sites (Figure $3 \mathrm{~A}$ ). In addition, the dual-luciferase reporter gene system results shows that SPRY1 was targeted by miR-187 (Figure 3 A). Next, miR-187 mimics and inhibitor were successfully transfected into the hippocampus of normal rats (Figure $3 \mathrm{~B}$ ). Further results show that SPRY1 was significantly decreased by overexpressed miR-187 and increased by downregulated miR187 (Figure $3 \mathrm{C}$ ). The PTEN/PI3K/AKT cascade was proved to be the downstream target of $S P R Y 1$, which was also shown to be regulated $\begin{array}{llll}\text { by miR-187 (Figure } 3 & \mathrm{C} \text { ). Besides, }\end{array}$ overexpressed miR-187 decreased the expression levels of PTEN and activated PI3K/AKT signal pathway, whereas knock-down of miR-187 had the opposite effects, which increased PTEN expression and inactivated PI3K/AKT signal pathway (Figure $3 \mathrm{C}$ ).

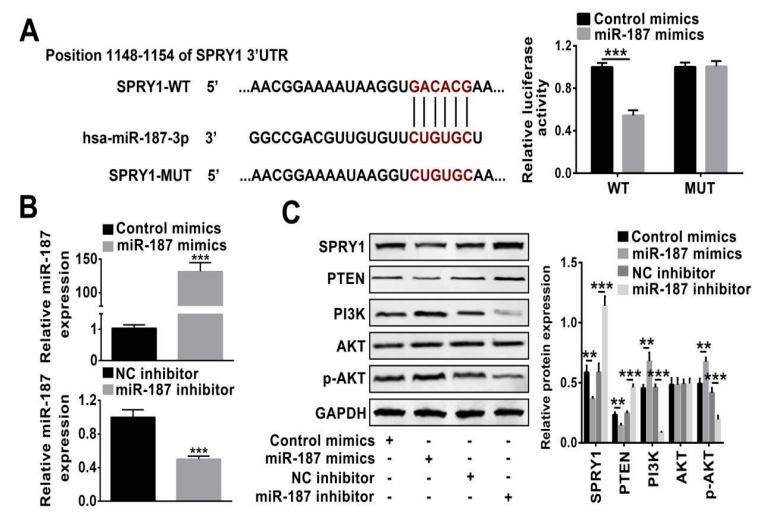

Figure 3: The interaction between SPRY1 and miR187. (A) TargetScan software was utilized to exploring the binding sites of miR-187 and SPRY1, and the dual-luciferase reporter gene system was applied to investigate the regulating mechanisms of miR-187 and SPRY1 in HEK-293T cells. (B) The transfection efficiency of miR-187 mimics and inhibitor were evaluated by RT-qPCR in the neuronal cells from epileptic rats. (C) The expression levels of SPRY1, PTEN, PI3K, AKT and p-AKT were evaluated by western blot. ${ }^{* *} p<0.05,{ }^{* \star *} p<0.01$, compared to the corresponding controls

MiR-187 regulates neuronal apoptosis and the development of epilepsy in PTZ-induced rat models by modulating SPRY1

SPRY1 participated in the regulation of cell apoptosis and has also been proved to be regulated by miR-187 in the experiments. Hence, this study next explored whether miR-187 regulates neuronal apoptosis and epilepsy development in rats by regulating SPRY1. First, this study successfully overexpressed SPRY1 in the neuronal cells of the rats (Figure $4 \mathrm{~A}$ and $\mathrm{B}$ ). The results show that the inhibiting effects of miR-187 on epilepsy development in PTZinduced rat models were abrogated by synergistically overexpressing SPRY1 (Figure 4 C). In addition, the inhibiting effects of overexpressed miR-187 on PTZ-induced neuronal apoptosis were also reversed by $S P R Y 1$ overexpression (Figure $4 \mathrm{D}$ and $\mathrm{E}$ ). 
A

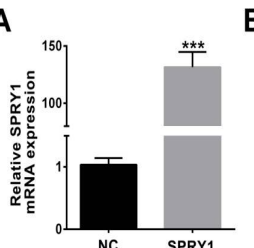

D

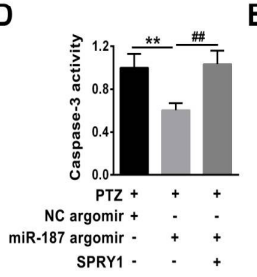

E
C $\rightarrow \mathrm{PTZ}+\mathrm{NC}$ argomir
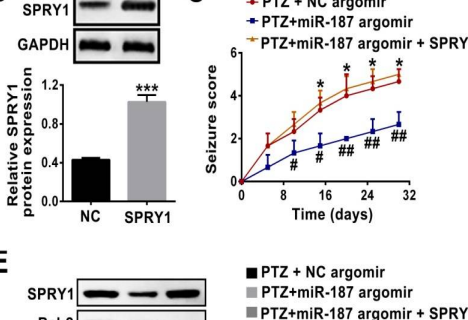

- PTZ + NC argomir -PTZ+miR-187 argomir

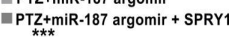

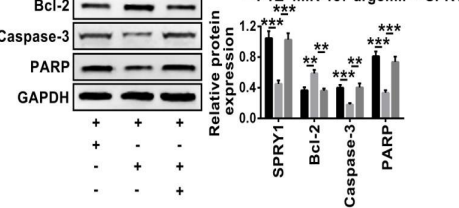

Figure 4: Effects of miR-187 on neuronal apoptosis and the development of epilepsy in epileptic rats $(A)$ SPRY1 mRNA and (B) protein levels in the hippocampus regions of PTZ-treated rats were evaluated to determine the transfection efficiency of $S P R Y 1$ overexpression vectors. (C) Seizure score of epileptic rats treated with miR-187 agomir, a SPRY1 overexpression vectors were evaluated according to the Racine's scale $(n=6)$. (D) Caspase 3 activity was evaluated in epileptic rats treated with miR-187 agomir and SPRY1 overexpression vectors. (E) The levels of BCL-2, caspase 3 and PARP were evaluated in epileptic rats treated with miR-187 agomir and SPRY1 overexpression vectors; ${ }^{* *} p<0.05,{ }^{* * *} p<0.01$, compared to the corresponding controls

\section{DISCUSSION}

Epilepsy is a chronic neurological disorder that greatly threatens human health [28], but the underlying mechanisms of the development of epilepsy are still unclear. Recent studies have showed that hippocampal neuronal apoptosis is critical for epilepsy [29]. Moreover, researchers reported that upregulation of Trem2 inhibits hippocampal neuronal apoptosis-mediated epilepsy via the PI3K/AKT signal pathway [29] and neuronal apoptosis-induced epilepsy in mice can be attenuated by sodium valproate [30]. These studies indicate that neuronal apoptosis is critical for epilepsy development and can be targeted to alleviate epilepsy.

MicroRNAs are small non-coding RNAs that contain an average of approximately 22 nucleotides [4] and function as gene regulators to modulate multiple genes expression. miRNAs have been reported to participate in the regulation of neuronal apoptosis [31] and epilepsy. MicroRNA-187 is a microRNA that has been reported to be closely related to epilepsy $[9,10]$. In addition, miR-187 has also been proved to regulate neuronal functions, including apoptosis, as well as neuronal development [33]. Hence, it is reasonable to speculate that miR-187 might be pivotal for neuronal apoptosis and epilepsy development. However, the detailed mechanisms are not fully delineated. The results showed that miR-187 was lowly expressed in the hippocampus resions of PTZ-treated rats. Furthermore, overexpressed miR-187 decreased PTZ-induced neuronal apoptosis and the development of epilepsy in rat models. These results are in accordance with previous studies $[9,10]$ which also reported the protective role of miR-187 in epilepsy.

Sprouty homolog 1 is located on chromosome 4 of human beings and has been reported to participate in the regulation of various cell functions [34]. In addition, SPRY1 has been reported to regulate neuronal apoptosis $[22,35]$ and predicted as the potential downstream target of miR-187 by using the TargetScan software, which indicated that miR-187 might alleviate PTZ-induced epilepsy in rats by targeting SPRY1. However, there are still no literature reports of a role of SPRY1 in the development of epilepsy.

Additional results show that overexpressed miR187 downregulated the expression levels of SPRY1 and PTEN. Similarly, knock-down of miR-187 upregulated SPRY1 and PTEN expression levels, which indicates that SPRY1 and PTEN could be downregulated by overexpressed miR-187. Furthermore, PTEN inactivated PI3K/AKT cascade [36] and was crucial for the regulation of epilepsy development [37]. This results found that overexpressed miR187 promoted the activation of PI3K/AKT signal pathway, which is in accordance with a previous study [38].

It is well known that miRNAs are pivotal for the regulation of cell functions [39]. This study found that miR-187 and SPRY1 are critical for neuronal apoptosis and epilepsy progression. Mechanistically, this study shows that upregulation of SPRY1 reverse the effects of overexpressed miR-187 on neuronal apoptosis and the development of epilepsy in PTZ-induced rat models. Taken together, miR-187 participates in the development of epilepsy by targeting SPRY1.

\section{CONCLUSION}

Overexpressed miR-187 inhibits neuronal apoptosis and the development of epilepsy in the hippocampus of PTZ-induced rat models by downregulating SPRY1 expression levels. These results can hopefully be helpful for the discovery of new therapeutic agents for epilepsy treatment in clinic. 


\section{DECLARATIONS}

\section{Acknowledgement}

This work was supported by China National Natural Sciences Foundation (Grant no. 81760809 Guangxi Natural Sciences Foundation Grant no. 2017GXNSFAA198294), Guangxi Health Technology and Development Foundation (Grant no. s2017049).

\section{Conflict of interest}

No conflict of interest is associated with this work.

\section{Contribution of authors}

We declare that this work was done by the researchers listed in this article. All liabilities related with the content of this article will be borne by the authors. Limei Diao, Haichun Yu and Xianqiang Qiu designed all the experiments and revised the paper. Huan $\mathrm{Li}$, Shungui Wang and Qian $\mathrm{Yu}$ performed the experiments, Huanxia Li, Ling Lu and Xianqiu Liao wrote the manuscript.

\section{Open Access}

This is an Open Access article that uses a funding model which does not charge readers or their institutions for access and distributed under the terms of the Creative Commons Attribution License (http://creativecommons.org/licenses/by/ 4.0) and the Budapest Open Access Initiative (http://www.budapestopenaccessinitiative.org/rea d), which permit unrestricted use, distribution, and reproduction in any medium, provided the original work is properly credited.

\section{REFERENCES}

1. Fisher RS, Acevedo C, Arzimanoglou A, Bogacz A, Cross $J H$, Elger CE, Engel J, Jr., Forsgren L, French JA, Glynn $M$ et al. ILAE official report: a practical clinical definition of epilepsy. Epilepsia 2014; 55(4): 475-482.

2. Maganti RK, Rutecki P. EEG and epilepsy monitoring. Continuum (Minneap Minn) 2013; 19(3 Epilepsy): 598622.

3. Guo XQ, Cao YL, Hao F, Yan ZR, Wang ML, Liu XW. Tangeretin alters neuronal apoptosis and ameliorates the severity of seizures in experimental epilepsyinduced rats by modulating apoptotic protein expressions, regulating matrix metalloproteinases, and activating the PI3K/Akt cell survival pathway. Adv Med Sci 2017; 62(2): 246-253.
4. Ambros $V$. The functions of animal microRNAs. Nature 2004; 431(7006): 350-355.

5. Karnati HK, Panigrahi MK, Gutti RK, Greig NH, Tamargo IA. miRNAs: Key Players in Neurodegenerative Disorders and Epilepsy. J Alzheimers Dis 2015; 48(3): 563-580.

6. Liu D, Li S, Gong L, Yang Y, Han Y, Xie M, Zhang $C$. Suppression of microRNA-141 suppressed p53 to protect against neural apoptosis in epilepsy by SIRT1 expression. J Cell Biochem 2019; 120(6): 9409-9420.

7. Tang $C$, Gu $Y$, Wang $H$, Wu $H$, Wang $Y$, Meng $Y$, Han $Z$, Gu $Y$, Ma W, Jiang $Z$ et al. Targeting of microRNA-21$5 p$ protects against seizure damage in a kainic acidinduced status epilepticus model via PTEN-mTOR. Epilepsy Res 2018; 144(34-42.

8. Tang L, He S, Zhu Y, Feng B, Su Z, Liu B, Xu F, Wang X, Liu H, Li C et al. Downregulated miR-187 contributes to the keratinocytes hyperproliferation in psoriasis. J Cell Physiol 2019; 234(4): 3661-3674.

9. Zhang $S$, Kou Y, Hu C, Han Y. MicroRNA profiling in the dentate gyrus in epileptic rats: The role of miR-187-3p. Medicine (Baltimore) 2017; 96(22): e6744.

10. Alsharafi WA, Xiao B, Abuhamed MM, Bi FF, Luo ZH. Correlation Between IL-10 and microRNA-187 Expression in Epileptic Rat Hippocampus and Patients with Temporal Lobe Epilepsy. Front Cell Neurosci 2015; $9(466$.

11. Mao XH, Chen $M$, Wang $Y$, Cui PG, Liu SB, Xu ZY. MicroRNA-21 regulates the ERK/NF-kappaB signaling pathway to affect the proliferation, migration, and apoptosis of human melanoma A375 cells by targeting SPRY1, PDCD4, and PTEN. Mol Carcinog 2017; 56(3): 886-894.

12. Lu Z, Wang F, Yu P, Wang $X$, Wang $Y$, Tang ST, Zhu $H Q$. Inhibition of miR-29b suppresses MAPK signaling pathway through targeting SPRY1 in atherosclerosis. Vascul Pharmacol 2018; 102(29-36.

13. Xia J, Wang $H$, Zhang $Q$, Han Z. Modulation of P2X Purinoceptor 3 (P2X3) in Pentylenetetrazole-Induced Kindling Epilepsy in Rats. Med Sci Monit 2018; 24(61656177.

14. Guerreiro CA. Epilepsy: Is there hope? Indian J Med Res 2016; 144(5): 657-660.

15. Liu AH, Chu M, Wang YP. Up-Regulation of Trem2 Inhibits Hippocampal Neuronal Apoptosis and Alleviates Oxidative Stress in Epilepsy via the PI3K/Akt Pathway in Mice. Neurosci Bull 2019; 35(3): 471-485.

16. Li Q, Li QQ, Jia JN, Cao S, Wang ZB, Wang X, Luo C, Zhou HH, Liu ZQ, Mao XY. Sodium Valproate Ameliorates Neuronal Apoptosis in a Kainic Acid Model of Epilepsy via Enhancing PKC-Dependent GABAAR gamma2 Serine 327 Phosphorylation. Neurochem Res 2018; 43(12): 2343-2352.

17. Zhang ZB, Tan YX, Zhao Q, Xiong LL, Liu J, Xu FF, Xu $Y$, Bobrovskaya L, Zhou XF, Wang TH. miRNA-7a-2-3p Inhibits Neuronal Apoptosis in Oxygen-Glucose Deprivation (OGD) Model. Front Neurosci 2019; 13(16. 
18. Subramanian $P$, Choi IC, Mani V, Park J, Subramaniyam S, Choi KH, Sim JS, Lee CM, Koo JC, Hahn BS. StageWise Identification and Analysis of miRNA from RootKnot Nematode Meloidogyne incognita. Int J Mol Sci 2016; 17(10).

19. Wang $P$, Zhou $Y$, Yang JQ, Landeck $L$, Min $M$, Chen $X B$, Chen JQ, Li W, Cai SQ, Zheng $M$ et al. The role of Sprouty 1 in the proliferation, differentiation and apoptosis of epidermal keratinocytes. Cell Prolif 2018; 51(5): e12477.

20. Gu X, Su X, Jia C, Lin L, Liu S, Zhang $P$, Wang $X$, Jiang $X$. Sprouty 1 regulates neuritogenesis and survival of cortical neurons. J Cell Physiol 2019; 234(8): 1284712864.

21. Yang X, Kilgallen S, Andreeva V, Spicer DB, Pinz I, Friesel R. Conditional expression of Spry 1 in neural crest causes craniofacial and cardiac defects. BMC Dev Biol 2010; 10 (48.
22. Zhao L, Yang XR, Han X. MicroRNA-146b induces the PI3K/Akt/NF-kappaB signaling pathway to reduce vascular inflammation and apoptosis in myocardial infarction by targeting PTEN. Exp Ther Med 2019; 17(2): 1171-1181.

23. Xiao Z, Peng J, Gan N, Arafat A, Yin F. Interleukin-1beta Plays a Pivotal Role via the PI3K/Akt/mTOR Signaling Pathway in the Chronicity of Mesial Temporal Lobe Epilepsy. Neuroimmunomodulation 2016; 23(5-6): 332344.

24. Cai Y, Ruan J, Yao X, Zhao L, Wang B. MicroRNA-187 modulates epithelial-mesenchymal transition by targeting PTRF in non-small cell lung cancer. Oncol Rep 2017; 37(5): 2787-2794.

25. Wang F, Zhang QW, Fu XH, Wan HF, Liu YLJTJoPR. Expressions and clinic significance of miRNA-143, miRNA- 34A, miRNA-944, miRNA-101 and miRNA-218 in cervical cancer tissues. 2016; 15(7): 1387-1392. 